\title{
One-Dimensional Magnonic Crystal With Cu Stripes for Forward Volume Spin Waves
}

\author{
Taichi Goto, ${ }^{1,2,3,{ }^{*}}$ Kei Shimada, ${ }^{1}$ Yuichi Nakamura, ${ }^{1}$ Hironaga Uchida, ${ }^{1}$ and Mitsuteru Inoue ${ }^{1}$ \\ ${ }^{1}$ Department of Electrical and Electronic Information Engineering, Toyohashi University of Technology, \\ 1-1 Hibarigaoka, Tempaku, Toyohashi, Aichi 441-8580, Japan \\ ${ }^{2}$ JST, PRESTO, 4-1-8 Honcho, Kawaguchi, Saitama 332-0012, Japan \\ ${ }^{3}$ Department of Materials and Science and Engineering, Massachusetts Institute of Technology, \\ 77 Massachusetts Avenue, Cambridge, Massachusetts 02139, USA
}

(Received 12 September 2018; revised manuscript received 7 December 2018; published 17 January 2019)

A one-dimensional magnonic crystal for forward volume spin waves (FV SWs) is demonstrated using eight pairs of $\mathrm{Cu}$ stripes fabricated on a $1-\mathrm{mm}$ wide $\times 14-\mathrm{mm}$ long $\times 10.2-\mu \mathrm{m}$ thick yttrium iron garnet (YIG) waveguide and a SW absorber of 30-nm-thick Au film. The development of this crystal is challenging due to strong spectral oscillations caused by edge reflections and process difficulties associated with YIG magnonic crystals. A magnonic band gap with a depth of $-4.6 \mathrm{~dB}$ is observed at a frequency of $1.80 \mathrm{GHz}$ for a FV SW excited by a $50-\mu$ m-wide microstrip line, which is in good agreement with simulation results using three-dimensional modeling in the radio frequency region. The obtained performance is illustrated by plotting the depth of the respective band gaps vs the pair number of the stripes. This value is compared with that obtained in previous studies.

DOI: 10.1103/PhysRevApplied.11.014033

\section{INTRODUCTION}

One dimensional (1D) magnonic crystals (MCs) for controlling the propagation of spin waves (SWs) have been widely studied and reviewed [1-13], primarily because of the unique properties of such crystals, e.g., the guiding and filtering of SWs [14-17], confinement of SWs [18-21], and slowing (phase shifting) of SWs. MCs are based on phase interference of SWs, and many applications have been proposed or are in development based on the advantages of MCs for controlling SWs. In particular, MCs can be used in SW integrated circuits (ICs), including logic gates [22-28]. SW ICs have attracted attention as next-generation computing elements that generate only a few Joules of heat during propagation. For these applications, forward volume SWs (FV SWs) are the most suitable mode because of their in-plane isotropy. FV SWs propagate in out-of-plane magnetized media without changing their wavelength, even in curved, oblique, or rectangular waveguides [22,24]. Hence, the use of phase interference

\footnotetext{
*goto@ee.tut.ac.jp
}

Published by the American Physical Society under the terms of the Creative Commons Attribution 4.0 International license. Further distribution of this work must maintain attribution to the author(s) and the published article's title, journal citation, and DOI. including MCs with FV SWs is significantly promising. However, only a few experimental studies [29] have researched MCs using FV SWs, although many experimental reports have described two modes - specifically, surface SWs (S SWs) [30-35] and backward volume SWs (BV SWs) [3,29,36-38]. This is mainly because of the significant noise in the FV SW configuration, which makes it difficult to observe a magnonic band gap (MBG) in experiments that employ low damping materials (yttrium iron garnets, YIG) as waveguides. In this study, we demonstrate a 1D MC for FV SW propagation in YIG film by introducing a noise suppression technique based on metallization $[22,39,40]$. The radio frequency (rf) powers used for exciting SWs in this study are in the linear region, and the SWs are dipolar SWs because the wavenumber is in the order of $\mathrm{mm}$ [8]. The observed MBG is analyzed using an rf simulation and compared with previous reports on MCs.

\section{SIMULATION AND DISCUSSION}

\section{A. YIG waveguide}

First, we calculate the single-mode propagation of FV SWs without periodic structure. A $10.2-\mu \mathrm{m}$-thick 14$\mathrm{mm}$-long YIG line is used as a SW waveguide in a three-dimensional (3D) simulator (CST Microwave Studio 2016 SP7) based on the finite integration technique (FIT) $[41,42]$. The parameters used in the simulation are the saturation magnetization $4 \pi M_{s}=1749 \mathrm{G}$, relative permittivity 
$\epsilon_{r}=15.3$, gyromagnetic ratio $\gamma=2.8 \mathrm{MHz} / \mathrm{Oe}$, and magnetic damping $\alpha=7.4 \times 10^{-4}$. The internal magnetic field $H_{\text {in }}=622$ Oe is perpendicularly ( $z$ direction $)$ applied to the YIG waveguide. This YIG is placed onto two microstrip lines (MSLs). $\mathrm{Cu}$ with a thickness of $18 \mu \mathrm{m}$, width of $50 \mu \mathrm{m}$, and conductivity $\sigma$ of $5.96 \times 10^{7} \mathrm{~S} / \mathrm{m}$ is used to fabricate the MSL. The MSL is placed onto a dielectric substrate $\left(\epsilon_{\mathrm{r}}=4.4\right)$ with a thickness $t_{d}=0.5 \mathrm{~mm}$, and the backside of the dielectric substrate is covered by $\mathrm{Cu}$ and set as ground level. These parameters are the same as those used in our prior simulation [40].

To ensure single-mode propagation of the FV SW and to maximize the input amplitude of the SW, the dispersion curve of the SW in the YIG waveguide is calculated before modeling using magnetostatic equations [8]. The wavevector in the width $(y)$ direction $k_{m}$ of the SW in the waveguide is quantized as

$$
k_{m}=\frac{m \pi}{w} \quad(\mathrm{~m}=0,1,2,3, \ldots),
$$

where $w$ is the width of YIG, and the $x$-directional wavevector $k$ is expressed with the thickness $(z)$ direction wavenumber $k_{z}$ as [43]

$$
k=\sqrt{k_{z}^{2}-k_{m}^{2}} .
$$

Using the boundary conditions [8], the dispersion curve is derived as follows:

$$
\begin{aligned}
& k=\sqrt{\left[\frac{1}{2 t_{\mathrm{YIG}} \sqrt{\mu}} \ln \frac{\left(\sqrt{\mu}-\tanh \left(k_{z} t_{d}\right)\right)(\sqrt{\mu}-1)}{\left(\sqrt{\mu}+\tanh \left(k_{z} t_{d}\right)\right)(\sqrt{\mu}+1)}\right]^{2}-\left(\frac{m \pi}{w}\right)^{2}} \\
& (m=0,1,2,3, \ldots),
\end{aligned}
$$

where $t_{\mathrm{YIG}}$ is the thickness of YIG $(=10.2 \mu \mathrm{m}) . \mu$ is the diagonal component of the permeability of YIG and is expressed as:

$$
\mu=1+\frac{\left(\gamma H_{\mathrm{in}}+i \alpha f\right)\left(\gamma \cdot 4 \pi M_{s}\right)}{\left(\gamma H_{\mathrm{in}}+i \alpha f\right)^{2}-f^{2}},
$$

where $f$ is frequency [8]. In Eq. (3), $k_{z} t_{d}$ is sufficiently large, hence $\tanh \left(k_{z} t_{d}\right) \approx 1$. Therefore, Eq. (3) can be converted to

$$
\begin{gathered}
k=\sqrt{\left(\frac{1}{t_{\mathrm{YIG}} \sqrt{\mu}} \ln \frac{\sqrt{\mu}-1}{\sqrt{\mu}+1}\right)^{2}-\left(\frac{m \pi}{w}\right)^{2}} \\
(m=0,1,2,3, \ldots) .
\end{gathered}
$$

Figure 1(a) shows a plot of Eq. (5) for $m=1$ and 2 at $f=1.8 \mathrm{GHz}$. When $w$ is between approximately 0.75 and approximately $1.25 \mathrm{~mm}$, a single-mode SW can be obtained. Hence, a width $w$ of $1 \mathrm{~mm}$ is used in all subsequent simulations and experiments. The wavenumber $k$ is $3.7578 \mathrm{~mm}^{-1}$ and the wavelength $\lambda$ is $1.672 \mathrm{~mm}$ for $w=1 \mathrm{~mm}$. The dispersion curves for $w=1 \mathrm{~mm}$ with various $m$ are shown in Fig. 1(b). A single mode is obtained in the frequency range between approximately 1.780 and approximately $1.815 \mathrm{GHz}$ for $m=1$. The spectral robustness is approximately $35 \mathrm{MHz}$, which is sufficiently large for the experiments.

Based on these estimations, the waveguide feature is determined and 3D simulations are conducted. Figures 1(c) and 1(d) show the calculated distribution of an absolute value of the $x$-directional magnetic-flux density $\left|b_{x}\right|$ as the amplitude of FV SW at the surface of the YIG waveguide for $w=1$ and $2 \mathrm{~mm}$, respectively. Disordered propagation is observed during multimode propagation $(w=2 \mathrm{~mm})$. MCs are based on phase interference; hence, such a disordered propagation hinders the exhibition of a MBG. However, in contrast, the 1-mm-wide waveguide exhibits a monotonically decaying amplitude relative to the direction of propagation. Hence, a single-mode propagation of FV SW is obtained with a 1-mm-wide YIG waveguide in simulation. (a)

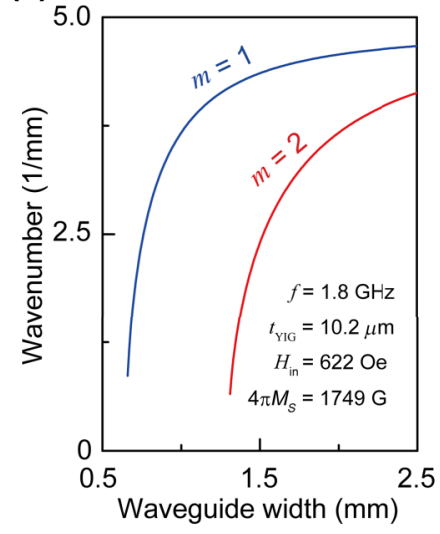

(b)

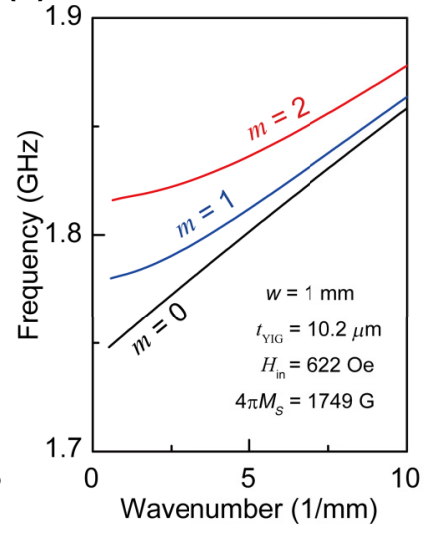

(c)

(d)

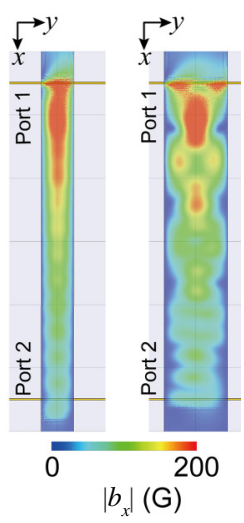

FIG. 1. (a) Calculated wavenumber as a function of the width of the YIG waveguide at a frequency of $1.8 \mathrm{GHz}$. (b) Dispersion curve of the FV SW at $w=1 \mathrm{~mm}$. (c),(d) Calculated amplitude distribution of the FV SW in YIG waveguides with widths of $w=1$ and $2 \mathrm{~mm}$. The thickness of the YIG $t_{\mathrm{YIG}}$ is $10.2 \mu \mathrm{m}$ and the saturation magnetization of $4 \pi M_{s}$ is $1749 \mathrm{G}$. The internal magnetic field in the YIG is $H_{\text {in }}=622$ Oe. 


\section{B. Modulation of the wavelength using $\mathrm{Cu}$}

We employ metallization techniques to modulate the wavelength of the FV SW for realizing MCs $[18,19,30,39,44,45]$. As shown in Ref. [39], the wavenumber $k_{\mathrm{Cu}-\mathrm{YIG}}$ of the FV SW propagation in $\mathrm{Cu}-\mathrm{YIG}$ is twice that in bare $k_{\mathrm{YIG}}(=k)$. Thus, at $f=1.8 \mathrm{GHz}$, the calculated wavenumbers are $k_{\mathrm{YIG}}=3.7578 \mathrm{~mm}^{-1}$ $\left(\lambda_{\mathrm{YIG}}=1.672 \mathrm{~mm}\right)$ and $k_{\mathrm{Cu}-\mathrm{YIG}}=1.8789 \mathrm{~mm}^{-1}$ $\left(\lambda_{\mathrm{Cu}-\mathrm{YIG}}=3.344 \mathrm{~mm}\right)$, respectively.

\section{Design of the magnonic crystal}

The pitch of the $\mathrm{Cu}$ stripes is determined using the following values. The length of the bare YIG area $L_{\mathrm{YIG}}$ is $418 \mu \mathrm{m}\left(=\lambda_{\mathrm{YIG}} / 4\right)$, the length of the $\mathrm{Cu}-\mathrm{YIG}$ area $L_{\mathrm{Cu}-\mathrm{YIG}}$ is $836 \mu \mathrm{m}\left(=\lambda_{\mathrm{Cu}-\mathrm{YIG}} / 4\right)$, and the eight pairs of $\mathrm{Cu}$ lines are introduced onto the YIG waveguide as shown in Fig. 2. The lattice constant is $1.254 \mathrm{~mm}$. The edges of the YIG waveguide outside the MSLs are covered by 30-nm-thick $\mathrm{Au}$ films, which function as SW absorbers for suppressing edge reflections and spectral noise $[22,39,40,46]$. The length of the $\mathrm{Au} L_{\mathrm{Au}-\mathrm{YIG}}$ is $1.8 \mathrm{~mm}$, the distance between the edges of the $\mathrm{Au}$ film and the edges of the MSL is $0.2 \mathrm{~mm}$, the conductivity of the Au is $4.56 \times 10^{7} \mathrm{~S} / \mathrm{m}$, and the position and thickness of the SW absorbers are as discussed and specified in Ref. [40].

The calculated transmission spectra of the FV SW with and without SW absorbers and with and without MCs are shown in Fig. 3. In Fig. 3(a), the YIG without the SW absorber does not exhibit distinct MBGs even with eight pairs of $\mathrm{Cu}$ strips because of the significant noise generated by the edge reflections. In contrast, the YIG with SW absorbers exhibits smooth transmission spectra, and the effect of MC, namely, distinct MBGs, is evident at $f=1.8 \mathrm{GHz}$. Hence, the SW absorbers are essential in order to observe MBGs in the FV SW. The estimated depth of the MBGs is about $-3.3 \mathrm{~dB}$.

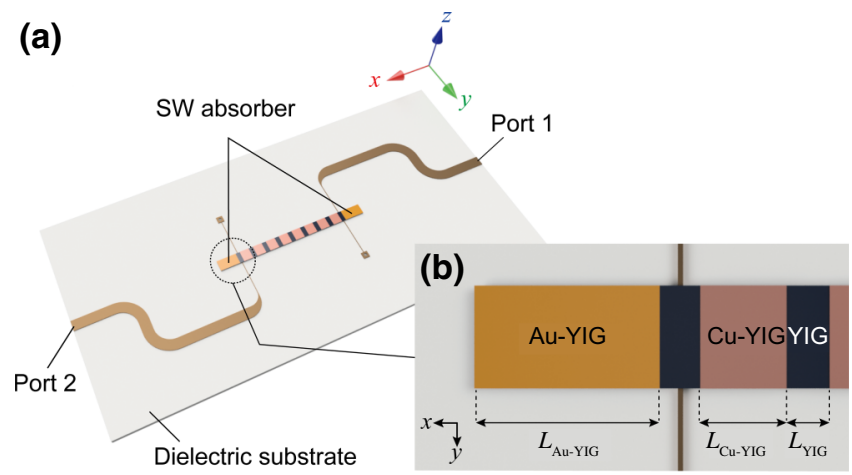

FIG. 2. 1D MC model containing eight pairs of $\mathrm{Cu}$ stripes. This model is used when simulating the FV SWs. (a) Overview of the simulation model and (b) an enlargement of the region close to the MSL and SW absorbers. The length of $\mathrm{Au}-\mathrm{YIG}, \mathrm{Cu}-\mathrm{YIG}$, and YIG are shown as $L_{\mathrm{Au}-\mathrm{YIG}}, L_{\mathrm{Cu}-\mathrm{YIG}}$, and $L_{\mathrm{YIG}}$, respectively. (a)

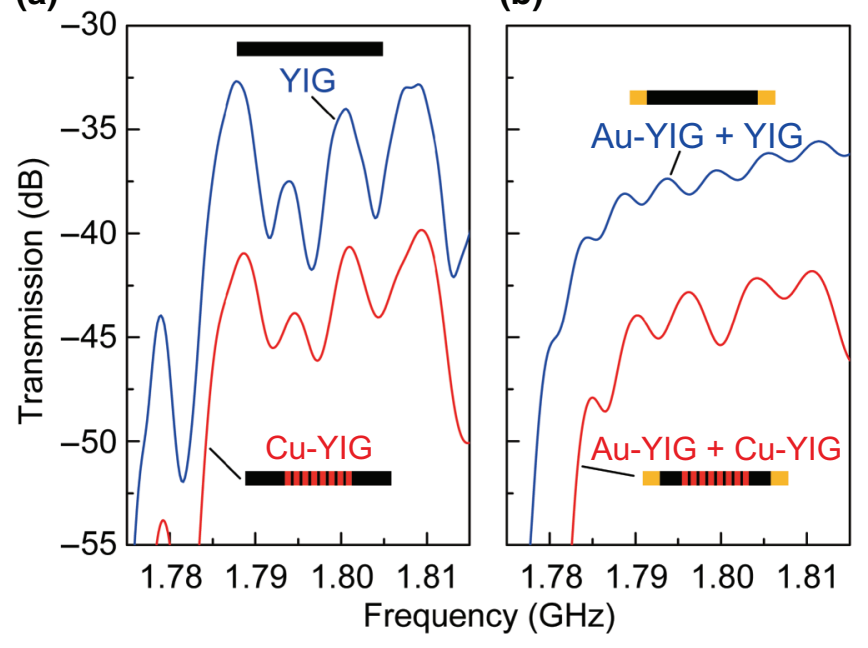

FIG. 3. (a) Calculated transmission spectra of the FV SW propagating in the YIG waveguide without SW absorbers and (b) with SW absorbers. The blue lines represent the transmission through the bare YIG waveguide, while the red lines indicate transmission through the YIG with the MC.

\section{EXPERIMENT AND DISCUSSION}

\section{A. Sample preparation}

An MC similar to the one simulated in the last section is lithographically prepared as shown in Fig. 4. A 10.2- $\mu \mathrm{m}-$ thick YIG grown on a gadolinium gallium garnet (GGG) substrate is cut into 1-mm-wide and 14-mm-long sections using a dicing saw (Disco, DAD321), as shown in Figs. 4(b) and 4(c). A 10-nm-thick layer of Ti is deposited

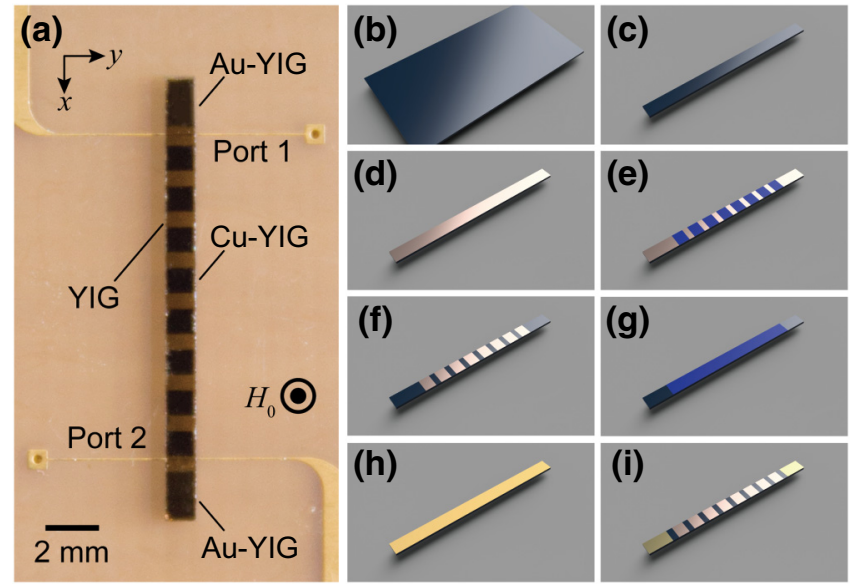

FIG. 4. Process for preparing the MC. (a) Overview of the fabricated sample. (b, c) The bare YIG is cut with a dicing saw. (d) $\mathrm{Cu}-\mathrm{Ti}$ is deposited onto the YIG. (e) The resist is exposed by a mask aligner. (f) The $\mathrm{Cu}-\mathrm{Ti}$ is etched. (g) Resist coating for SW absorbers. (h) Au-Ti deposition. (i) Lift-off step. 
by rf magnetron sputtering (Shimadzu, HSR551) under $20 \mathrm{mTorr} \mathrm{Ar}$ at a flow rate of $5 \mathrm{sccm}$ using a rf power of $70 \mathrm{~W}$ and a deposition rate of $6.7 \mathrm{~nm} / \mathrm{min}$. Next, $1-\mu \mathrm{m}-$ thick $\mathrm{Cu}$ is deposited by the same machine under $5 \mathrm{mTorr}$ Ar at a flow rate of $5 \mathrm{sccm}$ with a rf power of $100 \mathrm{~W}$ and a deposition rate of $50 \mathrm{~nm} / \mathrm{min}$ [Fig. 4(d)]. After sonication using acetone and isopropanol (IPA), the sample is baked at $170{ }^{\circ} \mathrm{C}$ for $2 \mathrm{~min}$. A $2-\mu \mathrm{m}$-thick layer of resist AZ5214 is spin coated onto the sample followed by baking at $100^{\circ} \mathrm{C}$ for $1 \mathrm{~min}$. The resist on the sample is exposed using a mask aligner (Canon, PLA501F) for $16 \mathrm{~s}$ followed

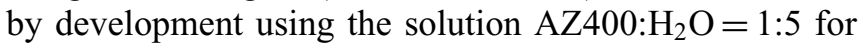
$200 \mathrm{~s}$ [Fig. 4(e)]. To etch the $\mathrm{Cu}$ and $\mathrm{Ti}$, the sample is dipped into $\mathrm{FeCl}_{3}$ for $5 \mathrm{~min}$ and $\mathrm{H}_{2} \mathrm{O}_{2}: \mathrm{NH}_{3}=50: 1$ for $5 \mathrm{~min}$. The remaining resist is removed by dipping into AZ700 for 5 min followed by cleaning using acetone and IPA without sonication [Fig. 4(f)]. The SW absorbers are then fabricated onto this sample using the same resist as above. After spin coating, exposure, development, and baking [Fig. 4(g)], a 30-nm-thick Au layer is deposited by the same magnetron sputtering machine under $3 \mathrm{mTorr} \mathrm{Ar}$ at a flow rate of $5 \mathrm{sccm}$ with a rf power of $40 \mathrm{~W}$ and a deposition rate of $16 \mathrm{~nm} / \mathrm{min}$, as shown in Fig. 4(h). After liftoff using AZ700 without sonication, the sample is cleaned with acetone and IPA [Fig. 4(i)]. The resulting sample is placed onto two MSLs-dielectric substrates [Flame Retardant Type 4 (FR4)], as shown in Fig. 4(a). The YIG surface is in contact with that of the MSLs. The sample is fixed by a ceramic sheet and nonmagnetic screws using a jig composed of brass. The two MSLs are connected to a vector network analyzer (VNA, Anritsu 37347C) via rf connectors. The bias magnetic field $H_{0}$ is perpendicularly applied to the sample using an electromagnet controlled using a proportional integral derivative (PID) method. The Gauss meter used in this setup is a F. W. Bell 7030 instrument and the current source is a Keysight N5750A. Transmission spectra are then obtained, followed by spin wave spectroscopy by varying the magnetic field.

\section{B. Observation of the magnonic band gap}

Figure 5(a) shows the obtained transmission spectra of the YIG waveguide with and without the MC. The bias magnetic field $H_{0}$ is $2.30 \mathrm{kOe}$. It can be seen that the simulated spectra shown in the previous section overlap. As per our design specifications, the MBG is generated at $f=1.80 \mathrm{GHz}$, which is in excellent agreement with the results of our simulation. The depth of the MBG is approximately $-4.6 \mathrm{~dB}$ in the experiment and approximately $-3.3 \mathrm{~dB}$ in the simulation. The difference between the experiment and simulation results may be due to the imperfectness of the sample and the modeling mesh-size limitation in the simulation. The large insertion loss is mainly due to an impedance mismatch at the $50-\mu \mathrm{m}$-wide MSLs. This can be improved in the future by optimizing the design of these antennas.

Figure 5(b) shows the FV SW spectroscopy image with varying magnetic fields from $2.25 \mathrm{kOe}$ to $2.35 \mathrm{kOe}$ with 0.5 -Oe steps. The observed kinks in the SW spectroscopy might be caused by the PID control error including the quantization error of an analog digital (AD) converter. In the red region, the FV SW is not excited. The blue area shows the propagation of the FV SW. Within this region, the right blue line corresponds to the observation and illustrates the MBG. The slope of this line is equal to the gyromagnetic ratio $\gamma$ of $2.8 \mathrm{MHz} / \mathrm{Oe}$; thus, this strongly indicates the obtained results are due to the FV SW. The many lines observed in the high-frequency region are due to higher modes generated by the MSL.

\section{Comparison and discussion}

The obtained depth of the MBG is compared with that of previous reports including those using the other two modes (S SW and BV SW), as shown in Fig. 6 and Table I. The color of the plots indicates the mode, and the shape of the plots indicates the type of structure used to realize the MCs. From the figure, it is evident that this study is one of (a)

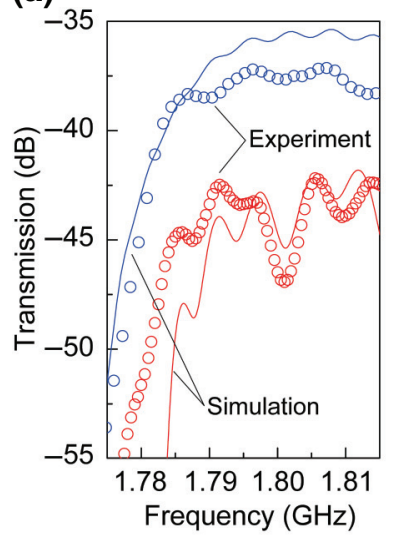

(b)

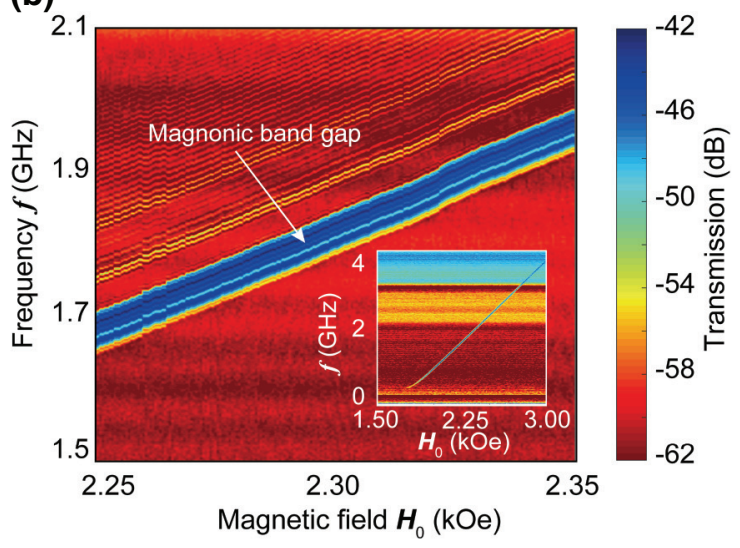

FIG. 5. (a) MBG for the FV SWs using an $\mathrm{MC}$ composed of $\mathrm{Cu}$ stripes on a YIG waveguide. The bias magnetic field $H_{0}$ is $2.30 \mathrm{kOe}$. The blue line and circles show the transmission spectra of the YIG waveguide. The red line and circles represent that of the YIG with the MC. (b) SW spectroscopy image of the MBG with a varying applied magnetic field $H_{0}$. The inset shows the wide range of the same spectra. 


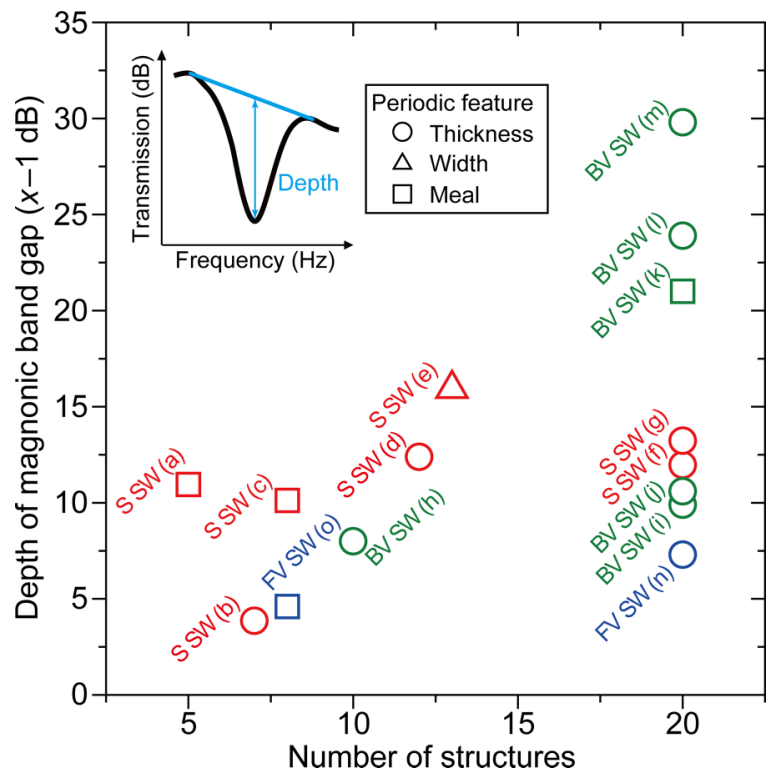

FIG. 6. Depth of the MBGs. The symbols in brackets correspond to the applicable reference shown in Table I. The red color represents the S SW, the green color represents the BV SW, while the blue color represents the FV SW. The circles indicate the MBGs using thickness modulation, the triangle indicates the MBGs using width modulation, and the squares indicate the same using metal stripes. This study is shown as (o).

a few reports on MCs for FV SW and the depth is comparable to that of the other modes. Because of the small number of $\mathrm{Cu}$-stripe pairs, the depth in the experiment at $-4.6 \mathrm{~dB}$ is not as large; however, this is similar to the results of the simulation. Hence, the increase in the number of stripes should increase the depth of the MBG.
In contrast, one can note that the only other report using FV SW, shown as (n) [29], does not show a large depth of MBG because that study focused only on the spectral position of MBG, not on depth. Hence, introducing the noise suppression technique and 3D-designing technique shown in this paper will increase the depth. In addition, Fig. 6 does not consider the observed frequency, applied field, properties of YIG, and measurement setup (i.e., the dynamic range of the network analyzer). This accounts for the large deviation displayed in the figure.

The simplest way to maximize the depth of MBG is to maximize the number of structures, and the maximum value can be determined by the propagation length of SWs. Therefore, to increase the depth of MBG, the suppression of insertion losses of the input and output ports using an impedance-matching technique and/or meander antenna should be effective. In addition, decreasing the damping factor of the SW waveguide material (including YIG) and its in-plane uniformity, and development of a precise nanoor micropatterning procedure for YIG would also help increase the propagation length of SWs and the depth of MBG.

\section{CONCLUSION}

A 1D MC is demonstrated using $\mathrm{Cu}$ stripes fabricated on $10.2-\mu \mathrm{m}$-thick YIG film based on FV SWs. This is a report of MBG for FV SWs using a periodically metallized YIG waveguide. The MBG is designed by combining magnetostatic equations and the FIT using a 3D model. At $f=1.80 \mathrm{GHz}$, a MBG with a depth of approximately $-4.6 \mathrm{~dB}$ is observed experimentally, which is close to the depth of $-3.3 \mathrm{~dB}$ observed in the simulation. The calculated results demonstrate the importance of

TABLE I. Comparison of the depth of the MBGs. All data are obtained from experimental reports shown with evaluable units. The number of structures means the number of metal lines, grooves, or a pair of notches in the waveguides. The depths show that of the first-order MBGs. The first-order MBGs for S SW and FV SW are observed at the lowest frequency, and those for BV SW are observed at the highest frequency.

\begin{tabular}{ccccccccc}
\hline \hline Symbol in Fig. 6 & Reference & Mode & Periodic feature & Number of structures & Depth $(\mathrm{dB})$ & $f(\mathrm{GHz})$ & $d(\mu \mathrm{m})$ & $H_{0}(\mathrm{kOe})$ \\
\hline (a) & {$[44]$} & S SW & Metal & 5 & -11.0 & 2.4 & 7.7 & 0.37 \\
(b) & {$[30]$} & S SW & Thickness & 7 & -3.9 & 4.8 & 10 & 1 \\
(c) & {$[30]$} & S SW & Metal & 8 & -10.1 & 4.9 & 10 & 1 \\
(d) & {$[32]$} & S SW & Thickness & 12 & -12.4 & 4.1 & 8.3 & 0.770 \\
(e) & {$[33]$} & S SW & Width & 13 & -15.9 & 2.5 & 10 & 0.444 \\
(f) & {$[35]$} & S SW & Thickness & 20 & -12.0 & 2.9 & 9 & 0.380 \\
(g) & {$[34]$} & S SW & Thickness & 20 & -13.2 & 7.2 & 5.5 & 1.845 \\
\hline (h) & {$[36]$} & BV SW & Thickness & 10 & -8.0 & 7.7 & 5.5 & 2.016 \\
(i) & {$[38]$} & BV SW & Thickness & 20 & -9.9 & 6.6 & 100 & 1.650 \\
(j) & {$[29]$} & BV SW & Thickness & 20 & -10.6 & 4.3 & 100 & 1.075 \\
(k) & {$[45]$} & BV SW & Metal & 20 & -21.0 & 6.5 & 5 & 1.80 \\
(1) & {$[34]$} & BV SW & Thickness & 20 & -23.9 & 7.2 & 5.5 & 1.845 \\
(m) & {$[3,37]$} & BV SW & Thickness & 20 & -29.8 & 7.2 & 5.5 & 1.845 \\
\hline (n) & {$[29]$} & FV SW & Thickness & 20 & -7.3 & 6.2 & 100 & 2.030 \\
(o) & This paper & FV SW & Metal & 8 & -4.6 & 1.8 & 10.2 & 2.300 \\
\hline \hline
\end{tabular}


the SW absorber in order to obtain a distinct MBG. This is an important component for controlling FV SWs when realizing SW ICs in the future.

\section{ACKNOWLEDGMENTS}

This work was partly supported by the following: JST PRESTO Grant No. JPMJPR1524 and JSPS KAKENHI Grants No. 17K19029, No. 16H04329, and No. 26220902. T.G. acknowledges the Yazaki memorial foundation of science and technology. We acknowledge Dr. Naoki Kanazawa, Mr. Takuya Yoshimoto, and Mr. Bungo Iwamoto for support and discussions. We also acknowledge Shin-Etsu Chemical Co., Ltd. for support.

[1] S. A. Nikitov, P. Tailhades, and C. S. Tsai, Spin waves in periodic magnetic structures - magnonic crystals, J. Magn. Magn. Mater. 236, 320 (2001).

[2] A. N. Kuchko, M. L. Sokolovskii, and V. V. Kruglyak, Spin wave spectrum of a magnonic crystal with an internally structured defect, Phys. B: Condens. Matter 370, 73 (2005).

[3] A. V. Chumak, A. A. Serga, B. Hillebrands, and M. P. Kostylev, Scattering of backward spin waves in a one-dimensional magnonic crystal, Appl. Phys. Lett. 93, 022508 (2008).

[4] A. V. Chumak, A. A. Serga, and B. Hillebrands, Magnonic crystals for data processing, J. Phys. D: Appl. Phys. 50, 244001 (2017).

[5] C. Banerjee, S. Choudhury, J. Sinha, and A. Barman, Pseudo-One-Dimensional Magnonic Crystals for HighFrequency Nanoscale Devices, Phys. Rev. Appl. 8, 014036 (2017).

[6] M. Krawczyk and D. Grundler, Review and prospects of magnonic crystals and devices with reprogrammable band structure, J. Phys.: Condens. Matter 26, 123202 (2014).

[7] B. Hillebrands and A. Thiaville, Spin dynamics in confined magnetic structures III (Springer-Verlag Berlin Heidelberg, New York, 2006).

[8] D. D. Stancil and A. Prabhakar, Spin Waves (Springer, New York, 2009).

[9] Z. K. Wang, V. L. Zhang, H. S. Lim, S. C. Ng, M. H. Kuok, S. Jain, and A. O. Adeyeye, Observation of frequency band gaps in a one-dimensional nanostructured magnonic crystal, Appl. Phys. Lett. 94, 083112 (2009).

[10] A. V. Chumak, T. Neumann, A. A. Serga, B. Hillebrands, and M. P. Kostylev, A current-controlled, dynamic magnonic crystal, J. Phys. D: Appl. Phys. 42, 205005 (2009).

[11] A. V. Chumak, P. Pirro, A. A. Serga, M. P. Kostylev, R. L. Stamps, H. Schultheiss, K. Vogt, S. J. Hermsdoerfer, B. Laegel, P. A. Beck, and B. Hillebrands, Spin-wave propagation in a microstructured magnonic crystal, Appl. Phys. Lett. 95, 262508 (2009).

[12] A. V. Chumak, V. I. Vasyuchka, A. A. Serga, and B. Hillebrands, Magnon spintronics, Nat. Phys. 11, 453 (2015).

[13] E. N. Beginin, A. V. Sadovnikov, A. Y. Sharaevskaya, A. I. Stognij, and S. A. Nikitov, Spin wave steering in three-dimensional magnonic networks, Appl. Phys. Lett. 112, 122404 (2018).

[14] S.-K. Kim, K.-S. Lee, and D.-S. Han, A gigahertz-range spin-wave filter composed of width-modulated nanostrip magnonic-crystal waveguides, Appl. Phys. Lett. 95, 082507 (2009).

[15] R. Zivieri, F. Montoncello, L. Giovannini, F. Nizzoli, S. Tacchi, M. Madami, G. Gubbiotti, G. Carlotti, and A. O. Adeyeye, Collective spin modes in chains of dipolarly interacting rectangular magnetic dots, Phys. Rev. B 83, 054431 (2011).

[16] K. Wagner, A. Kákay, K. Schultheiss, A. Henschke, T. Sebastian, and H. Schultheiss, Magnetic domain walls as reconfigurable spin-wave nanochannels, Nat. Nano 11, 432 (2016).

[17] M. Collet, O. Gladii, M. Evelt, V. Bessonov, L. Soumah, P. Bortolotti, S. O. Demokritov, Y. Henry, V. Cros, M. Bailleul, V. E. Demidov, and A. Anane, Spin-wave propagation in ultra-thin YIG based waveguides, Appl. Phys. Lett. 110, 092408 (2017).

[18] N. Kanazawa, T. Goto, and M. Inoue, Spin wave localization in one-dimensional magnonic microcavity comprising yttrium iron garnet, J. Appl. Phys. 116, 083903 (2014).

[19] N. Kanazawa, T. Goto, J. W. Hoong, A. Buyandalai, H. Takagi, and M. Inoue, Metal thickness dependence on spin wave propagation in magnonic crystal using yttrium iron garnet, J. Appl. Phys. 117, 17E510 (2015).

[20] F. S. Ma, H. S. Lim, V. L. Zhang, S. C. Ng, and M. H. Kuok, Magnonic band structure investigation of onedimensional bi-component magnonic crystal waveguides, Nanoscale Res. Lett. 7, 498 (2012).

[21] C. H. Chen, R. Z. Qiu, C. H. Chang, and W. J. Hsueh, Strongly localized modes in one-dimensional defect-free magnonic quasicrystals, AIP Adv. 4, 087102 (2014).

[22] N. Kanazawa, T. Goto, K. Sekiguchi, A. B. Granovsky, C. A. Ross, H. Takagi, Y. Nakamura, H. Uchida, and M. Inoue, The role of Snell's law for a magnonic majority gate, Sci. Rep. 7, 7898 (2017).

[23] M. Balynsky, A. Kozhevnikov, Y. Khivintsev, T. Bhowmick, D. Gutierrez, H. Chiang, G. Dudko, Y. Filimonov, G. Liu, C. Jiang, A. A. Balandin, R. Lake, and A. Khitun, Magnonic interferometric switch for multi-valued logic circuits, J. Appl. Phys. 121, 024504 (2017).

[24] S. Klingler, P. Pirro, T. Brächer, B. Leven, B. Hillebrands, and A. V. Chumak, Spin-wave logic devices based on isotropic forward volume magnetostatic waves, Appl. Phys. Lett. 106, 212406 (2015).

[25] T. Fischer, M. Kewenig, D. A. Bozhko, A. A. Serga, I. I. Syvorotka, F. Ciubotaru, C. Adelmann, B. Hillebrands, and A. V. Chumak, Experimental prototype of a spin-wave majority gate, Appl. Phys. Lett. 110, 152401 (2017).

[26] M. P. Kostylev, A. A. Serga, T. Schneider, B. Leven, and B. Hillebrands, Spin-wave logical gates, Appl. Phys. Lett. 87, 153501 (2005).

[27] K. Alexander, B. Mingqiang, and L. W. Kang, Magnonic logic circuits, J. Phys. D: Appl. Phys. 43, 264005 (2010).

[28] T. Schneider, A. A. Serga, B. Leven, B. Hillebrands, R. L. Stamps, and M. P. Kostylev, Realization of spin-wave logic gates, Appl. Phys. Lett. 92, 022505 (2008). 
[29] K. H. Chi, Z. Yun, M. Rongwei, S. Nikitov, Y. V. Gulyaev, and C. S. Tsai, Propagation characteristics of magnetostatic volume waves in one-dimensional magnonic crystals with oblique incidence, IEEE Trans. Magn. 47, 3708 (2011).

[30] V. D. Bessonov, M. Mruczkiewicz, R. Gieniusz, U. Guzowska, A. Maziewski, A. I. Stognij, and M. Krawczyk, Magnonic band gaps in YIG-based one-dimensional magnonic crystals: An array of grooves versus an array of metallic stripes, Phys. Rev. B 91, 104421 (2015).

[31] A. V. Sadovnikov, E. N. Beginin, M. A. Morozova, Y. P. Sharaevskii, S. V. Grishin, S. E. Sheshukova, and S. A. Nikitov, Nonlinear spin wave coupling in adjacent magnonic crystals, Appl. Phys. Lett. 109, 042407 (2016).

[32] C. L. Ordóñez-Romero, Z. Lazcano-Ortiz, A. Drozdovskii, B. Kalinikos, M. Aguilar-Huerta, J. L. Domínguez-Juárez, G. Lopez-Maldonado, N. Qureshi, O. Kolokoltsev, and G. Monsivais, Mapping of spin wave propagation in a onedimensional magnonic crystal, J. Appl. Phys. 120, 043901 (2016).

[33] S. E. Sheshukova, E. N. Beginin, A. V. Sadovnikov, Y. P. Sharaevsky, and S. A. Nikitov, Multimode propagation of magnetostatic waves in a width-modulated yttrium-irongarnet waveguide, IEEE Magn. Lett. 5, 1 (2014).

[34] A. V. Chumak, A. A. Serga, S. Wolff, B. Hillebrands, and M. P. Kostylev, Scattering of surface and volume spin waves in a magnonic crystal, Appl. Phys. Lett. 94, 172511 (2009).

[35] C. G. Sykes, J. D. Adam, and J. H. Collins, Magnetostatic wave propagation in a periodic structure, Appl. Phys. Lett. 29, 388 (1976).

[36] A. D. Karenowska, A. V. Chumak, A. A. Serga, J. F. Gregg, and B. Hillebrands, Employing magnonic crystals to dictate the characteristics of auto-oscillatory spin-wave systems, J. Phys.: Conf. Ser. 303, 012007 (2011).
[37] A. V. Chumak, A. A. Serga, S. Wolff, B. Hillebrands, and M. P. Kostylev, Design and optimization of onedimensional ferrite-film based magnonic crystals, J. Appl. Phys. 105, 083906 (2009).

[38] K. H. Chi, Y. Zhu, R. W. Mao, J. P. Dolas, and C. S. Tsai, An approach for analysis of magnetostatic volume waves in magnonic crystals, J. Appl. Phys. 109, 07D320 (2011).

[39] N. Kanazawa, T. Goto, K. Sekiguchi, A. B. Granovsky, C. A. Ross, H. Takagi, Y. Nakamura, and M. Inoue, Demonstration of a robust magnonic spin wave interferometer, Sci. Rep. 6, 30268 (2016).

[40] K. Shimada, T. Goto, N. Kanazawa, H. Takagi, Y. Nakamura, H. Uchida, and M. Inoue, Extremely flat transmission band of forward volume spin wave using gold and yttrium iron garnet, J. Phys. D: Appl. Phys. 50, 275001 (2017).

[41] T. Weiland, Time domain electromagnetic field computation with finite difference methods, Int. J. Numer. Model.: Electron. Netw. Devices Fields 9, 295 (1996).

[42] T. Weiland, M. Timm, and I. Munteanu, A practical guide to 3-D simulation, IEEE Microw. Mag. 9, 62 (2008).

[43] J. Adam and S. Bajpai, Magnetostatic forward volume wave propagation in YIG strips, IEEE Trans. Magn. 18, 1598 (1982).

[44] M. A. Morozova, S. V. Grishin, A. V. Sadovnikov, D. V. Romanenko, Y. P. Sharaevskii, and S. A. Nikitov, Band gap control in a line-defect magnonic crystal waveguide, Appl. Phys. Lett. 107, 242402 (2015).

[45] A. V. Chumak, V. S. Tiberkevich, A. D. Karenowska, A. A. Serga, J. F. Gregg, A. N. Slavin, and B. Hillebrands, All-linear time reversal by a dynamic artificial crystal, Nat. Comm. 1, 141 (2010).

[46] N. Kanazawa, T. Goto, K. Sekiguchi, A. B. Granovsky, H. Takagi, Y. Nakamura, and M. Inoue, Spin wave absorber generated by artificial surface anisotropy for spin wave device network, AIP Adv. 6, 095204 (2016). 